\title{
Innocampus Explora: Una aproximación multidisciplinar a la problemática ambiental
}

\begin{abstract}
José Moros Gregorio ${ }^{\text {e1 }}$, Alejandro Quílez Asensio ${ }^{\text {e2 }}$, Daniel Jiménez Romero ${ }^{\text {e2 }}$, Ana Blas Medina ${ }^{e 3}$, Isaac Giménez Escamilla ${ }^{e 3}$, Laura Amorós Hernández ${ }^{\text {e3 }}$, Lola Giner ${ }^{\text {e3 }}$, Ana Crespo ${ }^{\text {e4 }}$, Inés Lledó Mateo ${ }^{\text {e4 }}$, Elisa Fernández Girao ${ }^{\text {e5 }}$, Juan Rodríguez ${ }^{\text {e5 }}$, Ezequiel López Serrano ${ }^{\text {e6 }}$, Adrián Oliver Belando ${ }^{\text {e6 }}$, Dídac Cuenca Peris ${ }^{\text {e6 }}$, Ana Chirivella Juan ${ }^{\text {e6 }}$, Antonio Iorga ${ }^{\text {e6}}$, M. Luisa Cervera Sanz $^{\mathrm{p} 1}$, Carlos Vila Descals ${ }^{\mathrm{p} 1}$, Rafael Ibáñez Puchades ${ }^{\mathrm{p} 1}$, Rafael García Gil ${ }^{\mathrm{p} 2}$, Inmaculada García Robles ${ }^{\mathrm{pc} 3}$, Luís F. Pascual Calaforra ${ }^{\mathrm{pc} 3}$, Teresa Garrigues Pelufo ${ }^{\mathrm{p} 4}$, Javier Pereda Cervera

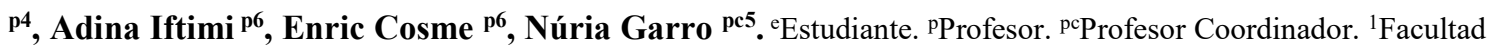
de Química. ${ }^{2}$ Escola Tècnica Superior d'Enginyeria (ETSE-UV). ${ }^{3}$ Facultad de Ciencias Biológicas. ${ }^{4}$ Facultad de Farmacia. ${ }^{5}$ Facultad de Física. ${ }^{6}$ Facultad de Matemáticas.
\end{abstract}

Correo institucional del proyecto: innocampus@uv.es.

\begin{abstract}
We present the activities of the Innocampus Explora innovation project developed on the Burjassot-Paterna campus of the Universitat de València and whose main objective is to show the interrelation between the different scientific and technical degrees on campus. In this year, the work team made up of students and professors from all the faculties and schools of the BurjassotPaterna campus, have carried out activities around environmental issues. A cross-sectional and interdisciplinary vision of the problems of the uses of plastic and nuclear energy that link with several of the Sustainable Development Goals (SDGs) dictated by the United Nations. With the development of this project we contribute to quality transversal training for all participating students.
\end{abstract}

Keywords: interdisciplinariety, science, disemination, interactivity, collaboration, environment

\section{Resumen}

Presentamos las actividades del proyecto de innovación Innocampus Explora desarrollado en el campus de Burjassot-Paterna de la Universitat de València y cuyo objetivo principal es mostrar la interrelación existente entre los diferentes grados científicos y técnicos del campus. En la presente anualidad, el equipo de trabajo integrado por estudiantes y profesores de todos las facultades y escuelas del campus de Burjassot-Paterna, ha desarrollado actividades en torno a la problemática mediambiental. Una visión transversal e interdisciplinar de los problemas de los usos del plástico $y$ de la energía nuclear que enlaza con varios de los Objetivos de Desarrollo Sostenible (ODS) dictados por Naciones Unidas. Con el desarrollo de este proyecto contribuimos a una formación transversal de calidad para todos los estudiantes participantes.

Palabras clave: Innovación, interdisciplinariedad, ciencia, divulgación, interactividad, colaboración, medio ambiente. 


\section{Introducción}

La interdisciplinariedad es un valor al alza en muchos campos del conocimiento, muy especialmente en el ámbito de las ciencias naturales y la tecnología. Existe una relación evidente entre el avance científicotécnico y la necesidad de combinar conocimientos y habilidades cada vez más específicas (Carvajal, 2010; Llano, 2016). Es por ello que desde las instituciones de educación superior deben implementarse mecanismos que promuevan, de manera activa, la relación entre los estudiantes de las diversas titulaciones y que contribuyan a fomentar la cultura de colaboración que resultará esencial para el desarrollo profesional de los egresados. El proyecto Innocampus Explora nace para servir de plataforma de unión y coordinación entre los centros del campus de Ciencias de la Universitat de València y su objetivo principal es organizar actividades divulgativas de carácter científico con un enfoque transversal que transmitan a los estudiantes de los grados de ciencias e ingeniería la cultura de la interdisciplinariedad. Por otro lado, el proyecto pretende poner en marcha un equipo de trabajo conformado por profesores y estudiantes de todos los centros que integran el campus de Burjassot-Paterna en el que, de una forma horizontal, rompiendo la jerarquía impuesta por el rol académico, se promueva la interacción y colaboración entre personas de las distintas titulaciones para implentar las actividades del proyecto desarrollando, con ello, nuevas habilidades y creando sinergias. Entre dichas actividades destacan especialmente aquellas relacionadas con la difusión usando nuevos canales y nuevas estrategias y en las que los estudiantes tienen un papel preponderante. La Universitat de València reconoce la labor de los estudiantes en el proyecto con 1,5 créditos a petición de las comisiones académicas de título de todos los grados involucrados en el proyecto.

Las titulaciones universitarias implicadas son: grado en Física, grado en Óptica y Optometría, grado en Química, grado en Biología, grado en Bioquímica y Ciencias Biomédicas, grado en Biotecnología, grado en Ciencias Ambientales, grado en Matemáticas, grado en Ciencia y Tecnología de los Alimentos, grado en Ciencias Gastronómicas, grado en Farmacia, grado en Nutrición Humana y Dietética, doble grado en Farmacia y en Nutrición Humana y Dietética, grado en Ingeniería Electrónica Industrial, grado en Ingeniería Electrónica de Telecomunicación, grado en Ingeniería Informática, grado en Ingeniería Multimedia, grado en Ingeniería Química, grado en Ingeniería Telemática y grado en Ciencia de Datos.

Innocampus Explora es un proyecto consolidado que ya marcha por su quinta edición y que cuenta con financiación por parte del Servei de Formació Permanent i Innovació Educativa (SFPIE) del Vicerrectorado de Políticas de Formación y Calidad Educativa de la Universitat de València además de una aportación por parte de todos los centros que participan.

En esta comunicación presentamos las actividades del proyecto Innocampus Explora en su última anualidad (curso 19-20), cuyo objetivo específico fue el dar una visión multidisciplinar a varias problemáticas ambientales, como son la proliferación del uso de plásticos y los beneficios y riesgos que entraña la energía nuclear. Este objetivo está fuertemente relacionado con varios de los Objetivos de Desarrollo Sostenible (ODS) dictados por la ONU: el objetivo 7 Energía asequible y no contaminante; el 11 Ciudades y comunidades sostenibles; el 13 Acción por el clima; y muy especialmente el 17 Alianzas para lograr los objetivos, en el que la aportación científico-técnica resulta imprescindible. El artículo está organizado de la siguiente manera: en la sección 2 se detallan los objetivos generales del proyecto Innocampus Explora; en la sección 3, dedicada al desarrollo de la innovación, se describe primeramente las actividades realizadas en anualidades anteriores, para después presentar las dos actividades que el grupo de trabajo ha desarrollado en el curso actual; posteriormente, en la sección 4, se presentan y discuten los resultados del proyecto, distinguiendo por un lado la valoración de las personas asistentes a las distintas actividades y, por otro, la autoevaluación realizada entre los estudiantes participantes en el proyecto; finalmente, en la sección 5 se exponen las conclusiones principales y las propuestas de mejora para futuras ediciones. 


\section{Objetivos}

Innocampus Explora presenta los siguientes objetivos:

- Demostrar a estudiantes de grados de ciencias e ingenierías la interrelación existente entre los mismos y la interdisciplinariedad profesional que se puede desarrollar en base a sus estudios.

- Mostrar la presencia de la ciencia en el día a día de las personas, planteando una visión analítica y científica en temas cotidianos.

- Divulgar conocimientos científicos y técnicos concretos, desde una visión multidisciplinar, simplificándolos para hacerlos comprensibles a estudiantes y profesionales de diversas ramas de la ciencia y la tecnología.

- Fomentar el trabajo y gestión de un equipo interdisciplinar, formado por estudiantes y profesores, rompiendo la barrera del rol establecido por el entorno educativo.

- Mostrar a los estudiantes que los conocimientos impartidos en grados diferentes al que cursan pueden resultar de gran utilidad en su desarrollo profesional.

- Servir de plataforma para todas aquellas iniciativas de los estudiantes encaminadas a fomentar la interdisciplinariedad.

\section{Desarrollo de la innovación}

\subsection{Historia del proyecto Innocampus Explora}

La primera edición de Innocampus Explora se pone en marcha en el curso 2015-16 con la creación de una comisión de trabajo con representantes del Personal Docente e Investigador de todos los centros del campus a la que se unieron una decena de estudiantes. Desde ese curso el proyecto cuenta con una cuenta de correo (innocampus@uv.es) y una página web institucional (https://www.uv.es/innocampus/es/innocampus.html, Fig. 1). Así mismo, los estudiantes crearon perfiles en Facebook, Instagram y Twitter y son los responsables de su mantenimiento.

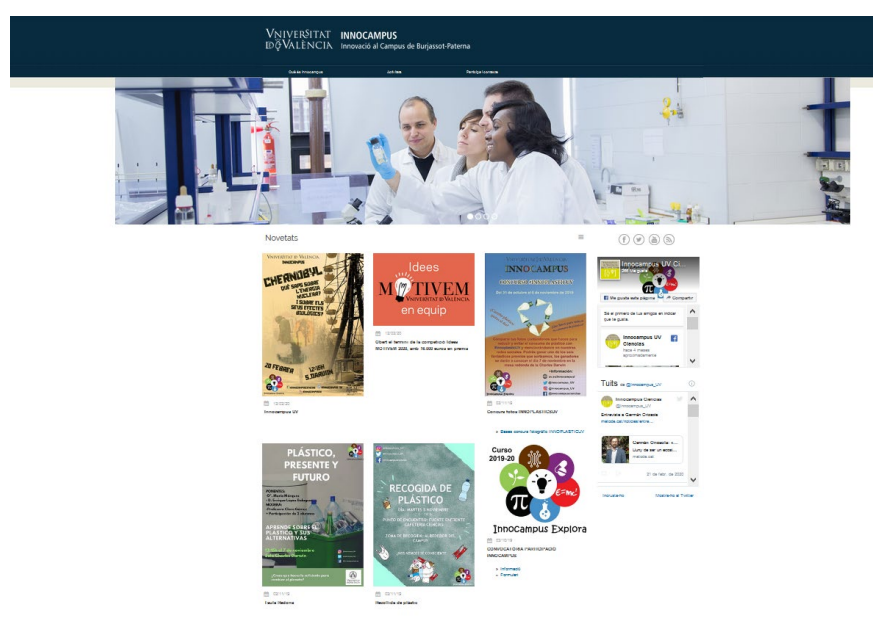

Fig. 1 Página web del proyecto Innocampus Explora.

En cada edición del proyecto se han organizado al menos dos actividades de difusión en forma de mesas redondas, fundamentalmente, aunque también se ha hecho uso de otros formatos como conferencias, 
monólogos, performances (ver Fig. 2), dirigidas a todos los estudiantes del campus. Por orden cronológico, estas han sido las actividades:

- La mesa redonda sobre "Nanociencia y Nanotecnología" (5 de mayo de 2016) que contó con la participación de profesores e investigadores de los diferentes centros e institutos de investigación del campus, quienes dieron una visión interdisciplinar y accesible de temas cercanos a su campo relacionados con el mundo de la nanotecnologia.

- La mesa redonda "La tierra más allá de la tierra" (20 de octubre de 2016) en la que estudiantes de los diferentes centros debatieron sobre los retos científico-tecnológicos que supondría la colonización de otro planeta. Analizaron el panorama científico actual en todo lo que implica un viaje espacial: relatividad, supervivencia durante periodos prolongados en el espacio, qué es posible encontrar, como emprender un viaje espacial, etc.

- La mesa redonda "Cerveza y ciencia" coordinada por los profesores (22 de marzo de 2017). En la misma se contó con la colaboración de empresas cerveceras artesanales valencianas que hicieron posible una cata de cerveza, así como con la ayuda de los alumnos de Ciencias Gastronómicas que prepararon un maridaje.

- Un concurso-certamen audiovisual que llevó por título "Concurso de ImágenesInterdisciplinariedad en la Ciencia” (marzo de 2017). En él los alumnos formaron grupos interdisciplinares de mínimo tres titulaciones a fin de realizar creaciones audiovisuales que expresaran el trabajo en grupo interdisciplinar. La entrega de premios fue el 5 de mayo de 2017 en el Salón de Grados de la Facultad de Matemáticas y las imágenes premiadas fueron expuestas de forma itinerante en el vestíbulo de los diferentes centros del campus y en Expociencia 2017, certamen abierto al público en general en el que científicos y emprendedores muestran experimentos, ideas y conocimientos de manera didáctica, interactiva y educativa.

- La mesa redonda “¿Es científico el cine?” (26 de octubre de 2017) en la que los ponentes fueron los propios estudiantes colaboradores del proyecto. Durante la misma, se proyectaron escenas de películas muy conocidas (aunque no siempre), al objeto de analizar, de forma interactiva, hasta qué punto el cine es científicamente riguroso. Los estudiantes de los centros del campus de Burjassot-Paterna pudieron debatir sobre los contenidos científicos presentados en las películas analizadas y a continuación se organizó una actividad interactiva, mediante la herramienta informática "Kahoot", de manera que los estudiantes participaron con sus móviles en una competición respondiendo a una serie de preguntas relacionadas con las escenas visualizadas. Resultó una actividad divertida en la que se pudo obtener conocimiento científico y también, cómo no, disfrutar del cine. Se entregó un obsequio a los 3 participantes que obtuvieron mayor puntuación.

- La mesa redonda "La ciencia... ¿con o contra el crimen?" (1 de marzo de 2018). Esta mesa contó con la intervención de profesores y profesionales que analizaron en qué medida la ciencia puede ayudar a cometer, pero también a resolver, actos delictivos. Se discutió si los conocimientos científicos que adquirimos en los diferentes grados científicos nos permiten acceder al ejercicio profesional contra el crimen en todas sus variantes. Nuevamente organizada por los alumnos miembros del proyecto, la mesa redonda contó con una competición interactiva mediante la herramienta informática "Kahoot". Se entregó un obsequio a los tres participantes que obtuvieron mayor puntuación.

- El concurso de fotografía "Interdisciplinariedad en la Ciencia" tuvo una segunda edición (marzo de 2018).

- La mesa redonda "Comunicar Ciencia" (8 de noviembre de 2018) en la que los estudiantes presentaron y debatieron sobre las distintas modalidades que actualmente se utilizan para 
comunicar ciencia: blogs, redes sociales, canales de youtube, literatura, cine, etc. En una segunda parte, se contó con la participación de los divulgadores de la "BigVan Ciencia" que presentaron dos monólogos científicos ampliamente celebrados.

- La proyección del documental "El enigma Agustina" de Manuel González y Emilio García (28 de febrero de 2019) seguido de un coloquio apoyado por una presentación audiovisual preparada por la organización, que se enfocó hacia las "otras Agustinas", las pioneras de la ciencia española que, en muchos casos fueron represaliadas tras la guerra civil y siempre poco reconocidas en su trabajo. Esta actividad nos ha permitido mostrar con un gran ejemplo, cómo el cine puede ser utilizado para comunicar ciencia siguiendo el camino recorrido por otros autores (Serrano, 2003; Gallego, 2007). Además, dada la temática y contenido de la película, también nos permitió tratar otros temas importantes como son el papel de la mujer en la ciencia española y la memoria histórica en relación con la actividad científica en nuestro país.
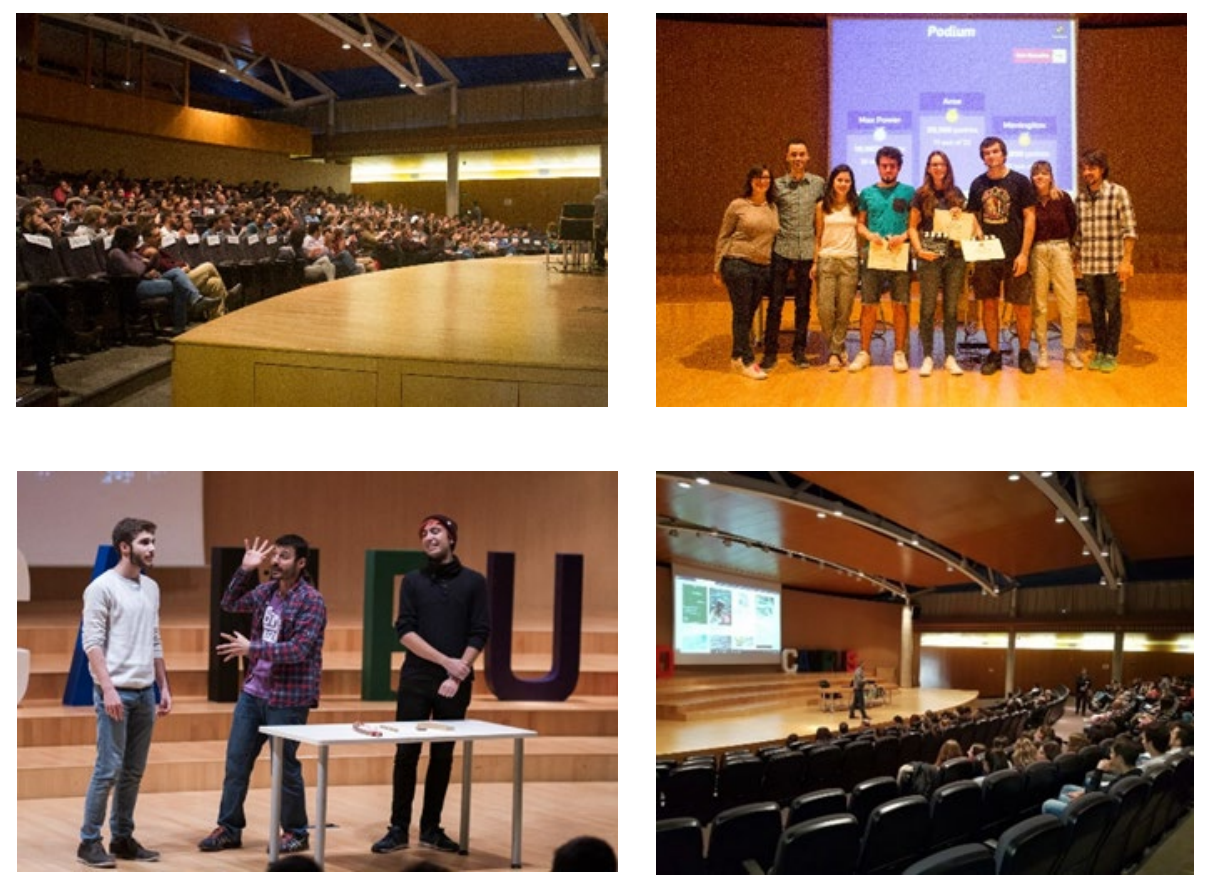

Fig. 2 Imágenes de las distintas actividades organizadas por Innocampus Explora. De izquierda a derecha y de arriba abajo: asistentes a la mesa redonda "Cerveza y Ciencia"; ganadores del kahoot "¿Es cientifico el cine?"; actuación de BigVan Ciencia; asistentes a la proyección del documental "El enigma Agustina".

Una de las tareas esenciales del proyecto es dar publicidad y visibilidad a las actividades realizadas. Para todas ellas, los estudiantes del proyecto han diseñado cuidadosamente y desarrollado la campaña de difusión. Ellos han sido los encargados de la producción de carteles (Fig. 3) o vídeos, así como su diseminación por redes sociales y otros canales.

A lo largo de estos años la coordinación del proyecto ha ido rotando entre representates de los distintos centros. Los sucesivos coordinadores/as han sido: la profesora Maria Luisa Cervera (Facultat de Química, curso 15-16); el profesor Javier Pereda Cervera (Facultad de Farmacia, curso 16-17); el profesor Juan Bautista Ejea (Escuela Técnica Superior de Ingeniería, curso 17-18); la profesora Inmaculada García 
Robles (Facultat de Biologia, curso 18-19); y en el curso actual la profesora Núria Garro (Facultat de Física).
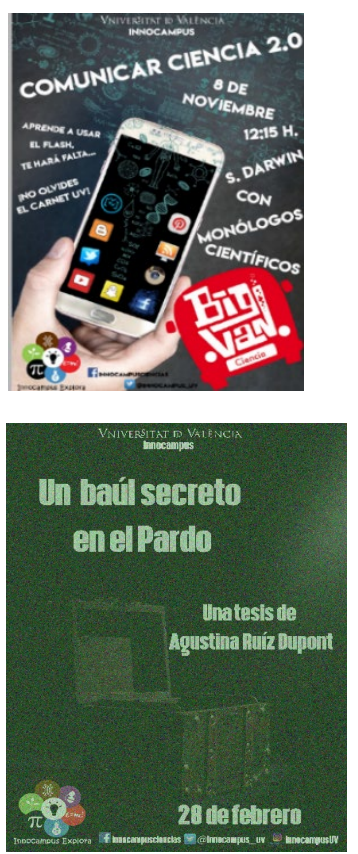
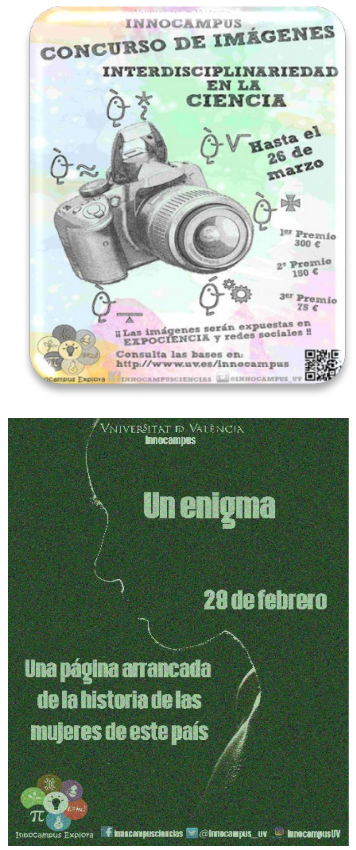
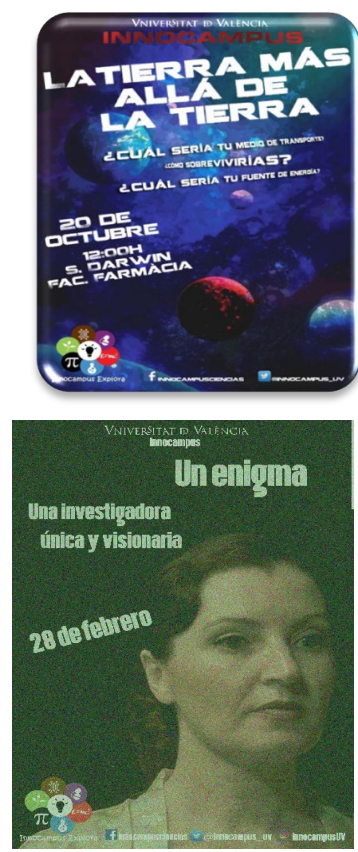

Fig. 3 Carteles promocionales de la mesa redonda "Comunicar ciencia" con la actuación de BigVan Ciencia, del concurso de fotografias "Interdisciplinariedad en la Ciencia” y de la mesa redonda "La Tierra más allá de la Tierra”. En la parte inferior, campaña de promoción de la proyección del documental "El enigma Agustina".

\subsection{El Proyecto en 2019-20}

Durante el presente curso, el Proyecto Innocampus Explora ha continuado su actividad focalizada en la visualización de la interdisciplinariedad en ciencia tomando, está vez, como hilo conductor la problemática medioambiental. Esta temática está implicada en varios de los Objetivos de Desarrollo Sostenibles (ODS) de la ONU, como son: el objetivo 7 Energía asequible y no contaminante; el 11 Ciudades y comunidades sostenibles; el 13 Acción por el clima; el 14 Vida submarina; el 15 Vida en ecosistemas terrestres. Por otro lado, el enfoque interdisciplinar que se da a todos los temas desde el proyecto incide directamente en el objetivo 17 Alianzas para lograr los objetivos.

El primer conjunto de actividades del presente curso se organizaron a lo largo de la primera semana de noviembre de 2019. La temática elegida fue el uso del plástico y toda la problemática que conlleva en la gestión de residuos. Para involucrar y concienciar a los estudiantes de los distintos grados del campus, se acunó el hastag \#InnoplasticUV y se hizo campaña en redes sociales (Fig. 4). Como parte de dicha campaña de concienciación, los estudiantes del proyecto hicieron varios videos cortos que se proyectaron durante toda la semana en las pantallas de las facultades y las cafeterías del campus. Otra de las actividades complementarias de la semana fue la recogida de plásticos y colillas en el campus y sus alrededores por parte de estudiantes voluntarios. La semana concluyó con la mesa redonda "Plástico: presente y futuro" organizada el jueves 7 de noviembre en la sala Darwin y que contó con dos ponentes: una investigadora del centro tecnológico de estudio del plástico Ainplast, y un representante del departamento de recogida de residuos del Ajuntament de València (Fig. 5). La actividad fue seguida por 
cerca de 140 asistentes, la mayoría de los cuales la encontraron bastante (45\%) o muy interesante (45\%). Al final del acto, se hizo un sorteo de obsequios entre todas las personas que habían participado en la recogida de plásticos.
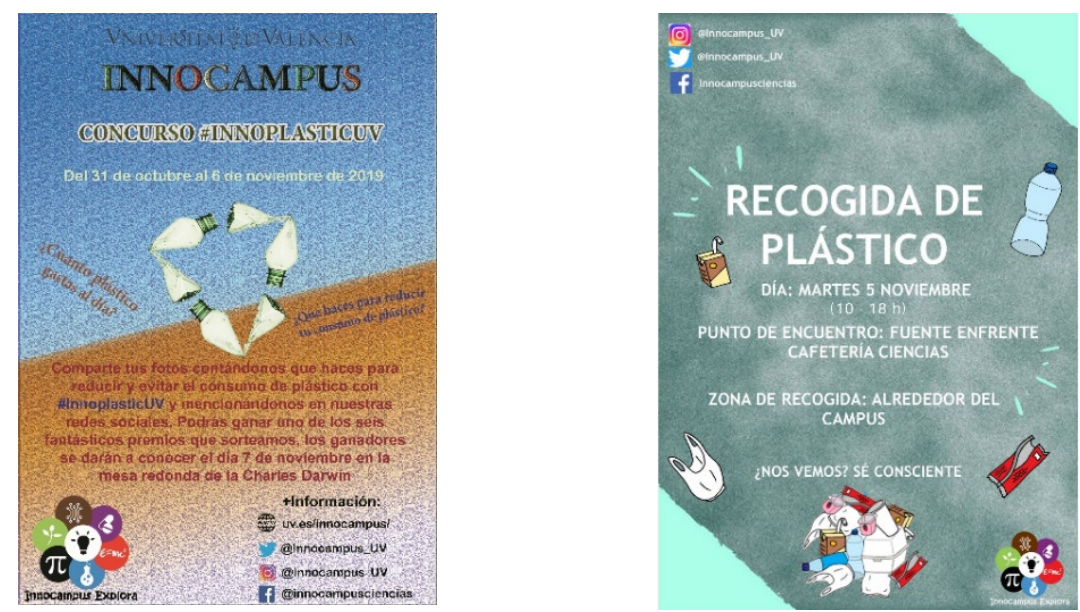

Fig. 4 Carteles de las actividades complementarias programdas en torno a la problemática medioambiental del plástico.

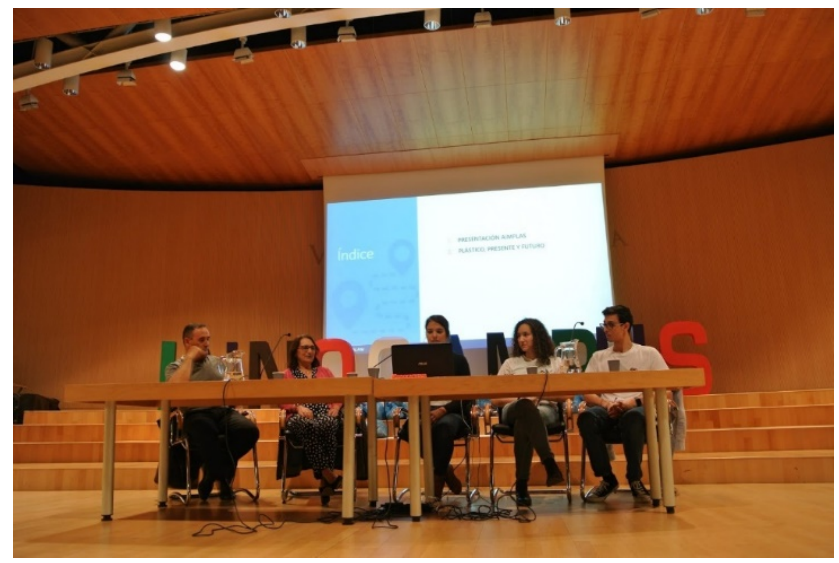

Fig. 5 Ponentes de la mesa redonda "Plástico: presente y futuro" acompañados por estudiantes del proyecto y la moderadora del acto .

La segunda actividad del curso trató sobre la energía nuclear, sus usos y sus potenciales peligros. Para centrar más el tema se planteó una mesa redonda con expertos en el tema titulada "Chernobyl: ¿Qué sabes de la energía nuclear?" que se realizó el 20 de febrero de 2020. La campaña de difusión, diseñada nuevamente por los estudiantes del equipo de trabajo, aprovechó la estética de la aclamada serie de TV "Chernobyl" para la realización de carteles (Fig. 6). Al inicio del acto se hizo una breve introducción a la radiactividad ambiental a cargo de la directora del Museo de Historia Natural de la Universitat de València, que también cedió parte de su colección de minerales, así como detectores de radiación para hacer una demostración. El panel de expertos contó con la presencia de un físico especializado en Física Nuclear, un biólogo experto en procesos ecológicos y evolutivos de organismos que viven en ambientes extremos y un profesor de Farmacia con amplia experiencia en radiofármacos. La actividad fue un gran éxito en cuanto a asistencia, rozando el límite del aforo de la sala Darwin (Fig. 6) y también en cuanto a valoración. 

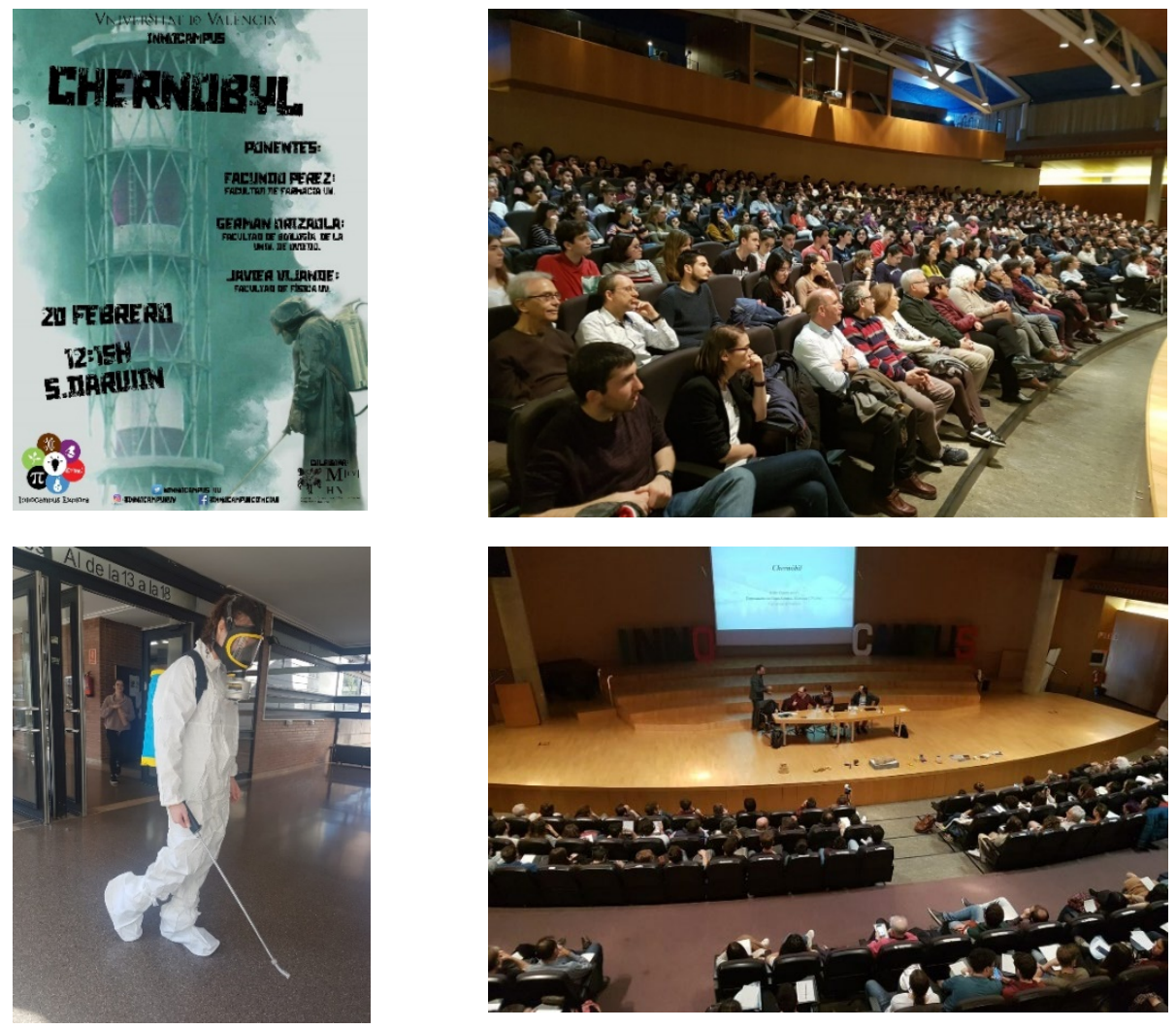

Fig. 6 En la parte izquierda, campaña de difusión de la mesa redonda "Chernobyl: ¿qué sabes sobre la energía nuclear?". En la parte derecha, imágenes del acto realizado en la sala Darwin, con aforo para 350 personas, el 20 de febrero de 2020.

\section{Análisis de los resultados}

El análisis de los resultados se ha realizado a tres niveles: en primer lugar analizamos la repercusión de las actividades dentro de la comunidad universitaria en base al número de personas asistentes en cada una de ellas; por otro lado, medimos el grado de consecución de los objetivos analizando las encuestas de satisfacción realizadas entre los asistentes; finalmente, valoramos la implantación de las metodologías del trabajo interdisciplinar entre los miembros del equipo de trabajo del proyecto.

\subsection{Repercusión de las actividades entre la comunidad universitaria del Campus}

La serie histórica de la asistencia a las distintas actividades del proyecto Innocampus Explora realizadas a lo largo de los distintos cursos se muestra en la Figura 7. Se observa que en los primeros años del proyecto se consiguió que la asistencia fuera creciendo en sucesivas actividades, lo cual permitió consolidar el proyecto Innocampus Explora entre el colectivo de estudiantes y profesores del campus. No obstante, en el curso pasado 18/19, habíamos detectado un decrecimiento persistente en el número de asistentes. Es por ello que entre los objetivos y la metodología de la presente edición, el equipo buscaba revertir esta tendencia incidiendo en las temáticas elegidas y, muy especialmente, en las campañas de difusión. Como puede observarse en la Figura 7, las dos útimas mesas redondas han conseguido mejorar las marcas del curso anterior, destacando especialmente la actividad dedicada a Chernobyl que ha sido, 
con diferencia, la más seguida desde el inicio del proyecto. Constatamos, por tanto, que el interés de los problemas medioambientales es transversal a las distintas ramas científicas y técnicas del campus y que ha sido un éxito en aras a mostrar la necesidad de los enfoques multidisciplinares.

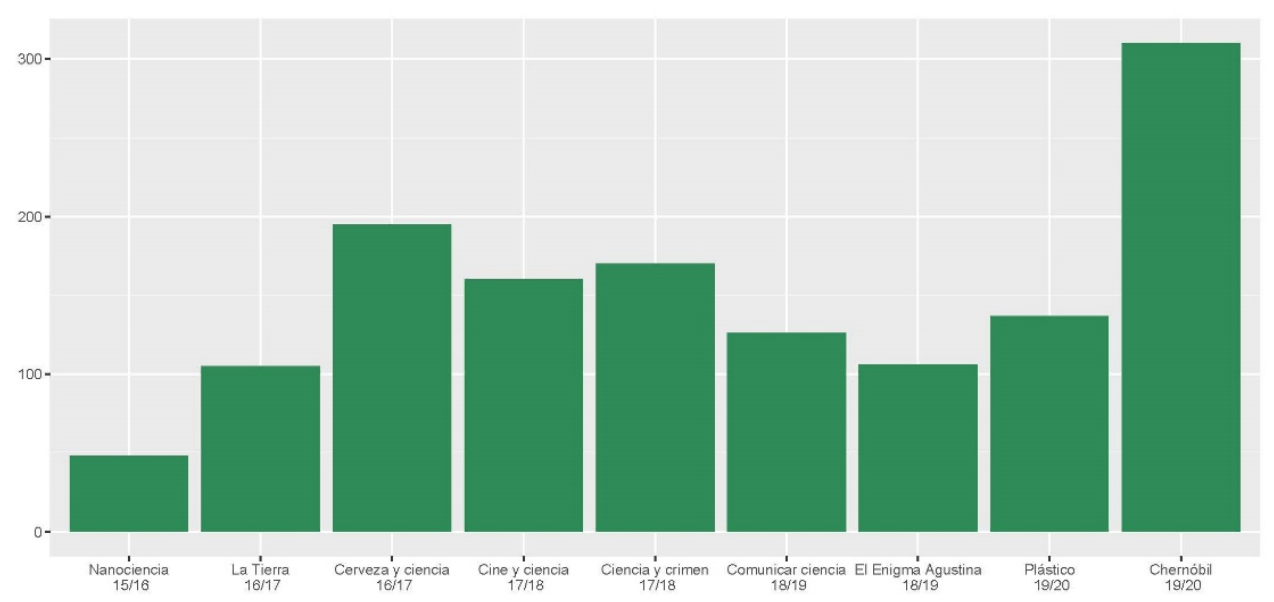

Fig. 7 Gráfica de asistencia a las diferentes actividades llevadas a cabo (en número de personas).

\subsection{Grado de satisfacción de los asistentes}

Se diseñó una encuesta para cuantificar la aceptación de las dos actividades realizadas en la presente anualidad del proyecto que se pasó a un número estadísiticamente representativo de los asistentes. Las preguntas de la encuesta (ver Fig. 8) incidían sobre la función de Innocampus para la difusión de la interdisciplinaridad, los contenidos y duración de la actividad, la organización y el interés despertado por la actividad. Las valoraciones son, en general muy positivas en todas las preguntas. La adecuación de las actividades al objetivo del proyecto de fomentar la interdisciplinariedad se valora como positiva o muy positiva con un porcentaje por encima del $80 \%$ como media. También la organización de la actividad recibe una aceptación similar. Respecto al grado de interés de las actividades relizadas este año, cerca del $90 \%$ de los asistentes lo han considerado alto o muy alto. El porcentaje de los asistentes que no ha visto satisfechas sus expectativas iniciales es inferior al 1\%. La duración de la actividad ha sido el aspecto que recibe una aceptación ligeramente menor, bajando hasta el 25\% (muy alto) y 50\% (alto). Reconocemos que el formato de mesa redonda, siendo el idóneo para la discusión multidisciplinar de un tema, suele resultar excesivamente largo en su duración que, en ambas actividades, excedió las dos horas. 
Plástico presente y futuro

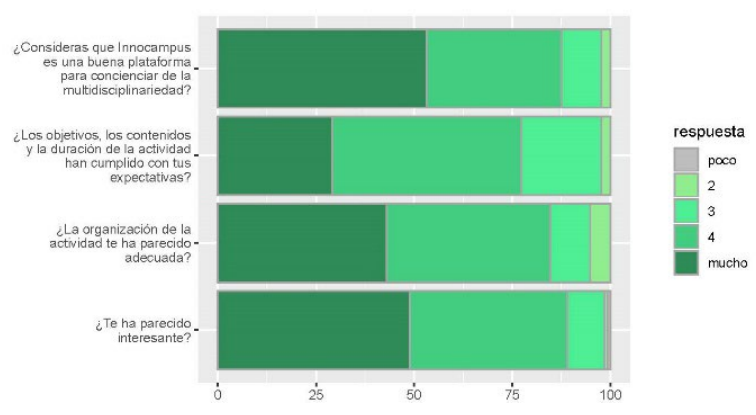

Chernobyl ¿qué sabes sobre la energía nuclear?

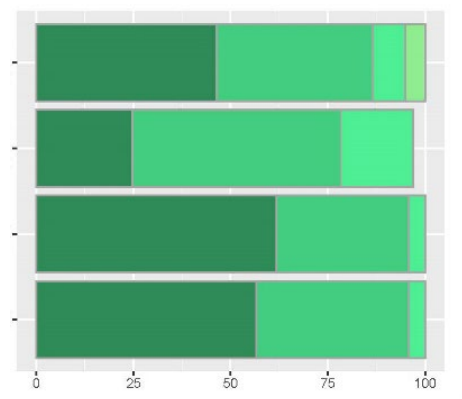

Fig. 8 Valoración de la adecuación de las actividades realizadas y de la idoneidad del proyecto para concienciar sobre la importancia de la interdisciplinariedad obtenidas tras las dos actividades realizadas a lo largo del curso 19/20: Mesas redondas "Plástico: presente y futuro” y “Chernobyl ¿qué sabes sobre la energía nuclear?”.

\subsection{Grado de satisfacción entre los integrantes del equipo de trabajo}

Finalmente, también se diseñó una encuesta de satisfacción para valorar el cumplimiento del objetivo de fomentar el trabajo y la gestión de un equipo interdisciplinar, integrando a estudiantes y profesores que se pasó entre los integrantes del equipo de trabajo. Los resultados de la encuesta revelan una valoración muy positiva de la experiencia del proyecto entre todos sus integrantes, tanto profesores como estudiantes. Entrando en más detalle, son los estudiantes los que muestran una valoración más crítica sobre las dinámicas de trabajo. Como se muestra en la Figura 9, cerca del 23\% de los estudiantes considera que el funcionamiento del equipo de trabajo es mejorable, mientras que un $78 \%$ lo considera bueno pero nadie lo califica de excelente. Preguntados sobre la viabilidad del proyecto de cara al futuro, la opinión mayoritaria está condicionada por una mayor implicación del equipo de trabajo (44\%) o por un cambio de objetivos y metas (11\%). Solamente el $11 \%$ consideró que no era viable. La encuesta también profundizaba en la valoración de la adquisición de distintas competencias: capacidad de análisis y síntesis; trabajo en un equipo interdisciplinar; conocimientos de informática relativos al ámbito de estudio; desarrollo de habilidades interpersonales; y conocimiento de los ODS. De los resultados se infiere que los estudiantes se muestran más satisfechos con el desarrollo de su capacidad para integrarse en un equipo multidisciplinar y las habilidades interpersonales. Por el contrario, se manifiestan poco satisfechos en su capacidad de análisis y sus conocimientos de la temática de los ODS en relación al Proyecto. 
El funcionamiento del equipo de trabajo en su conjunto te ha parecido: 9 respuestas

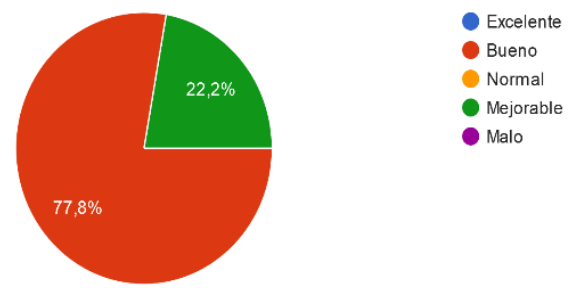

¿Ves futuro al proyecto?

9 respuestas

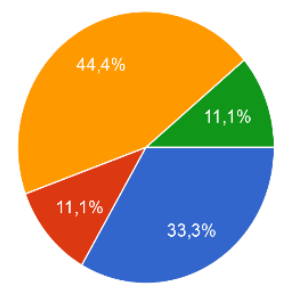

Si

No

Tal vez, con mayor implicación de

equipo de trabajo

Tal vez, con diferentes objetivos y metas

Fig. 9 Valoración de los estudiantes participantes en el proyecto sobre el funcionamiento del equipo de trabajo en su conjunto y el proyecto en futuras anualidades.

\section{Conclusiones}

En base a los resultados obtenidos podemos obtener algunas conclusiones interesantes, y plantear propuestas de mejora, relacionadas con tres aspectos fundamentales del proyecto como son $(i)$ el logro de los objetivos, (ii) la respuesta de la comunidad universitaria y (iii) el funcionamiento del equipo de trabajo.

El objetivo principal el proyecto es visualizar la importancia de la interdisciplinariedad en la ciencia y la tecnología y, dados los resultados, consideramos que estamos contribuyendo significativamente a una formación transversal de calidad tanto de los estudiantes que participan de las actividades programadas como, y especialmente, de los estudiantes que a lo largo de los distintos cursos participan en el desarrollo del proyecto Innocampus Explora como miembros del equipo de trabajo. La repercusión del proyecto, en relación con el seguimiento de las actividades programadas, ha sido muy positiva en la presente edición. El reto para futuras ediciones del proyecto estará en mantener las elevadas asistencias a las actividades, siendo innovadores en las campañas de difusión y acertando en el interés de las temáticas.

En cuanto al funcionamiento del grupo, aunque el grado de compromiso y colaboración entre profesores y estudiantes ha ido aumentando con los años, sigue habiendo margen de mejora. Siendo un colectivo grande y heterogéneo, hay una dificultad intrínseca en conseguir dinámicas óptimas y la experiencia acumulada durante estos años está siendo esencial para corregir los problemas que se han ido presentando. En cualquier caso destacamos el buen grado de satisfacción de los estudiantes del proyecto respecto al trabajo realizado, las competencias desarrolladas para la organización de las actividades y la interacción transversal e interdisciplinar dentro del equipo. 


\section{Referencias}

CARVAJAL, Y. (2010). "Interdisciplinariedad: desafio para la educación superior y la investigación" en Luna Azul, n. 31 p. $156-169$.

$<$ http://www.scielo.org.co/pdf/luaz/n31/n31a11.pdf $>$ [Consulta 21 de marzo de 2019]

GAlleGO, C. (2007). Tiem(pos)Modernos. Ensayos de Tecnociencia y cine. Madrid: Editorial Equipo Sirius, S. A.

LlANO, L., GUTIÉRREZ, M., STABLE, A., NÚÑEZ, M., MASÓ, M. Y ROJAS, B. (2016). "La interdisciplinariedad: una necesidad contemporánea para favorecer el proceso de enseñanza aprendizaje" en Medisur, v.14 n. 3, p. 320-327.

$<$ http://www.medigraphic.com/cgi-bin/new/resumen.cgi?IDARTICULO=66798> [Consulta 14 de marzo de 2019]

SERRANO, J. M. (2003). De lo fantástico a lo real. Diccionario de la ciencia en el cine. España: Editorial Nivola.

El enigma Agustina. (Dir. M. González y E. García). Instituto de Astrofísica de Andalucia, 2018.

$<$ https://www.iaa.csic.es/noticias/se-estrena-el-enigma-agustina-pelicula-producida-por-el-instituto-astrofisicaandalucia-iaa $>$ [Consulta 17 de enero de 2019]

Web del proyecto: www.uv.es/innocampus

Redes sociales: Twitter: @Innocampus_UV

Facebook:@innocampuscien > 